\title{
Analysis of Brain Natriuretic Peptide Serum Levels in Patients with Symptomatic Chronic Subdural Hematoma: A Potential Reliable Biomarker
}

\author{
Mehdi Chihi, Oliver Gembruch, Marvin Darkwah Oppong, Moritz Helsper, Bernd-Otto Hütter, \\ Ramazan Jabbarli, Karsten H. Wrede, Ulrich Sure, and Homajoun Maslehaty*
}

\begin{abstract}
The purpose of this study was to analyze brain natriuretic peptide (BNP) serum levels of patients with chronic subdural hematoma (cSDH) and their clinical implication. Patients with cSDH who underwent surgery in our department between November 2016 and October 2019 were eligible for enrollment in the study. Patients with recurrent bleedings, traumatic brain injury, cSDH associated with other intracranial pathologies, and those with a history of congestive heart failure, renal or endocrine disease were excluded. We measured BNP serum levels pre- and post-operatively and at discharge. The BNP values were analyzed with respect to patient medical history and neurological condition. The Glasgow Coma Scale score and the modified Rankin Scale score classified the clinical and neurological condition at the time of admission and discharge, respectively. The data of 100 surgically treated patients with cSDH (mean age 73.2, range $42-94$ years, male/female 3.5:1) were analyzed. Pre-operative BNP serum levels (BNP-1) were elevated in $67 \%$ of the patients $(n=67$; median $=101.6 \mathrm{pg} / \mathrm{mL} ; p<0.001)$. These serum levels increased after surgery $(\mathrm{p}<0.001)$ and decreased thereafter $(p<0.001)$, reaching a level at discharge (day 7) that was not statistically different from BNP-1 $(p>0.05)$. In addition, elevated BNP-1 showed a significant statistical association with the presence of atrial fibrillation $(p<0.01)$ and antiplatelet and/or anticoagulant therapy $(p<0.01)$. This study provides new evidence regarding BNP serum levels and their secretion pattern in patients with cSDH. Whether BNP-1 can predict the long-term functional outcome of patients with cSDH is being investigated in this ongoing prospective study.
\end{abstract}

Keywords: biomarker; brain natriuretic peptide; cardiac insufficiency; chronic subdural hematoma

\section{Introduction}

B RAIN NATRIURETIC PEPTIDE (BNP) is a potent, natriuretic, vasorelaxant peptide ${ }^{1}$ that is structurally similar to porcine $\mathrm{BNP}$, which was isolated originally from the porcine brain. ${ }^{2}$ The BNP is synthesized, stored and released mainly in the ventricular myocardium. ${ }^{3}$ The secretion of BNP is stimulated by the increase of volume or pressure. ${ }^{4}$ Expressed in the heart, BNP may reach the hypothalamus through the subfornical organ via the activation of the natriuretic receptor-A. ${ }^{1}$ Previous radioimmunoassay studies have detected BNP in brain regions including the hypothalamus and the subfornical organ. ${ }^{5}$

The BNP has a central role in the modulation of cardiovascular homeostasis, mainly through its inhibitory effect on the release of antidiuretic hormone, as well as in regulating the function of the hypothalamic-pituitary-adrenal axis. ${ }^{6}$ The BNP plays an important role in the prediction of congestive heart failure (CHF) in the acute phase and is accepted as a reliable biomarker. ${ }^{7}$ Recent studies, however, have found an increase of BNP in the acute phase of stroke, ${ }^{8-10}$ traumatic brain injury (TBI), ${ }^{11-13}$ aneurysmal subarachnoid hemorrhage (aSAH), ${ }^{14-18}$ and hypertensive intracerebral hemorrhage $(\mathrm{ICH})^{19}$; some studies have assessed the pattern of BNP secretion in some of these central nervous system (CNS) diseases. ${ }^{14,19}$ Regarding the interaction of BNP serum levels and cranial pathologies, the role of BNP has not yet been analyzed in patients with subdural hematoma (SDH), and more specifically in those with chronic SDH (cSDH).

Chronic SDH is seen frequently in neurosurgical centers and occurs predominately in the elderly population. ${ }^{20}$ It is the result of a mild head trauma that leads to the tearing of the bridging veins

Department of Neurosurgery and Spine Surgery, University Hospital Essen, Essen, Germany.

*Current address: Department of Orthopedics and Trauma Surgery, St-Vinzenz-Hospital, Dinslaken, Germany.

(C) Mehdi Chihi et al., 2020; Published by Mary Ann Liebert, Inc. This Open Access article is distributed under the terms of the Creative Commons Attribution Noncommercial License (http://creativecommons.org/licenses/by-nc/4.0/) which permits any noncommercial use, distribution, and reproduction in any medium, provided the original author(s) and the source are credited. 
and/or arachnoidea and leakage of blood and/or cerebrospinal fluid (CSF) in the subdural space. This leads to the formation of a small acute SDH and/or hygroma. ${ }^{21}$ Spontaneous resolution of both fluid accumulations is common because of highly active fibrinolytic systems contained in the $\mathrm{CSF}^{22}$ unless there is sufficient subdural space because of advanced age or cortical atrophy that results in a delay in absorption and formation of a neomembrane enclosing the hematoma. ${ }^{21}$
Because of the inflammatory and angiogenic responses that lead to microhemorrhages and fluid exudation through new fragile capillaries of the hematoma membrane, ${ }^{23}$ the hematoma continues to grow until symptoms occur. When a mass effect results in clinical symptomatology, surgical management is considered as the preferred treatment option. ${ }^{24}$

We hypothesized that the increasing brain compression from hematoma growth, which may lead to the elevation of the intracranial

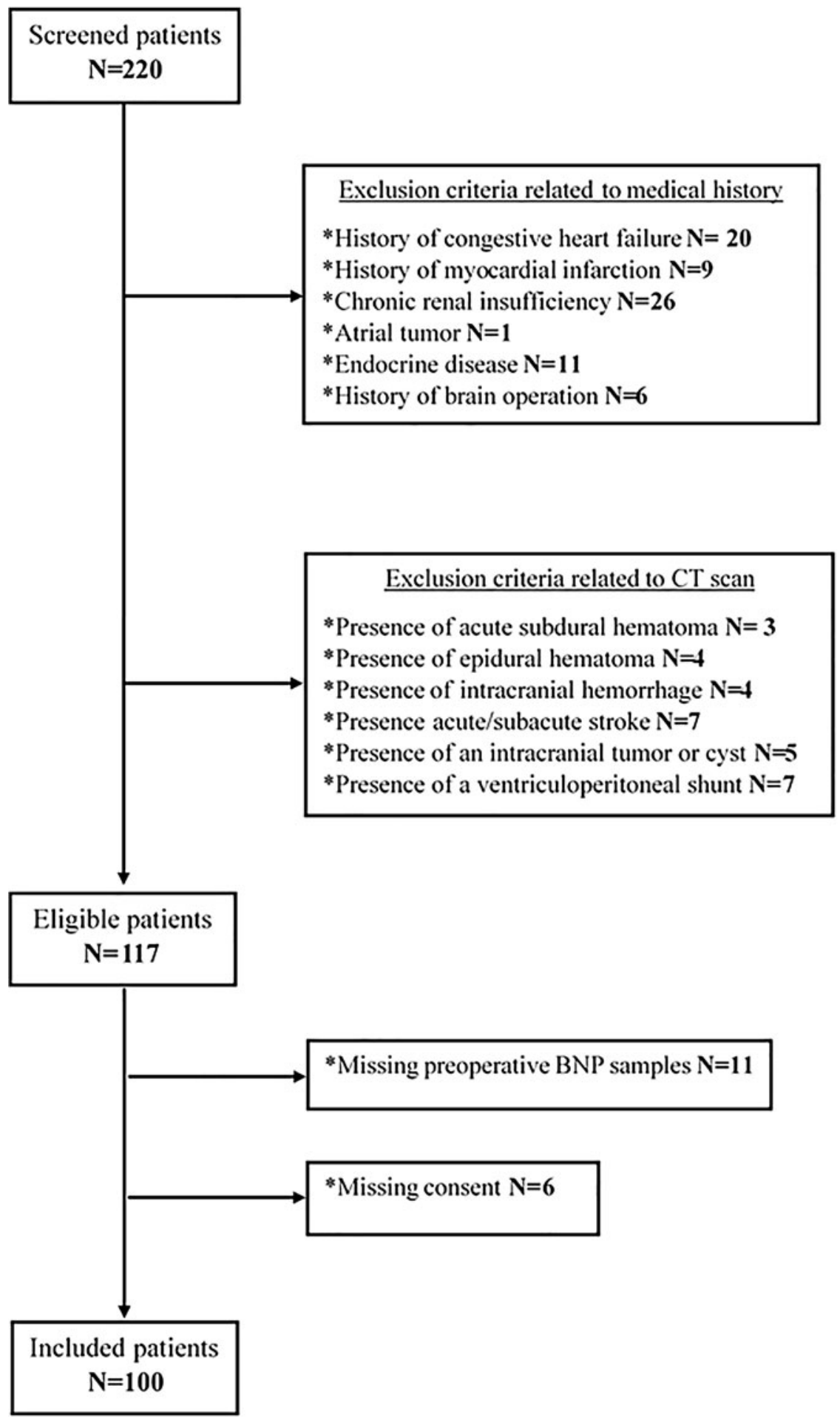

FIG. 1. Flow diagram of study inclusion and exclusion criteria. 
pressure (ICP), might be the cause of the increase in BNP serum level, this relationship between ICP and BNP being demonstrated recently in patients with $\mathrm{TBI}^{12,13}$ and hypertensive $\mathrm{ICH}{ }^{19}$

Thus, the purpose of the current study was to investigate the patterns of BNP serum levels in patients with symptomatic cSDH and their potential clinical implication.

\section{Methods}

\section{Study population}

All procedures performed in this study were in accordance with the ethical standards of the Institutional Research Committee as well as with the Declaration of Helsinki. Written informed consent was obtained from all patients. The Institutional Review Board approved this study (Medical Faculty, University of DuisburgEssen, Registration number: 15-6632-BO).

In the present single-center prospective study, we screened 220 patients ( $\geq 18$ years) with symptomatic $\mathrm{cSDH}$, who underwent surgical removal of the hematoma in our neurosurgical department between November 2016 and October 2019. We defined the term symptomatic as progressive headache, neurological impairments, seizures, and changes in behavior.

We excluded patients with a history of CHF $(n=20)$, history of myocardial infarction $(n=9)$ renal insufficiency $(n=26)$, endocrine disease $(n=11)$ at admission, presence of an atrial tumor $(n=1)$, history of brain operation $(n=6)$, presence of a concomitant acute SDH $(n=3)$, epidural hematoma $(n=4)$, ICH $(n=4)$, acute/subacute stroke $(n=7)$, presence of an intracranial tumor or cyst $(n=5)$, and presence of a ventriculoperitoneal shunt system $(n=7)$. A further 17 patients were excluded from analysis because of missing consent $(n=6)$ and incomplete pre-operative blood samples $(n=11)$. In total, 100 patients met the inclusion criteria and were analyzed in the current study. A flow diagram is presented in Figure 1.

\section{cSDH treatment protocol}

The diagnosis of cSDH was made by cranial computed tomography (CCT, Somatom Definition AS, Siemens Healthcare GmbH, Erlangen, Germany) before surgery in all cases. Hematoma evacuation was performed according to local policy with a single or widened burr-hole trepanation and insertion of a subdural drain for $48 \mathrm{~h}(n=91)$. Some patients, however, did not require any drainage $(n=3)$ or underwent operation through a craniotomy $(n=4)$ or a decompressive craniectomy $(n=2)$ without insertion of subdural drainage.

Peri-operative antibiotic prophylaxis was ensured by a single injection administrated before surgery (usually with cefazolin). Post-operatively, patients remained in the intermediate care unit for $48 \mathrm{~h}$. A control CCT was performed on the second post-operative day, and the subdural drain was removed. Patients were discharged usually on day 6 or day 7 after surgery. Routine blood samples were collected three times per week to control the salt-water balance. Patients with hyponatremia were treated initially intravenously, followed by oral intake.

\section{Serum BNP sample collection and management}

In total, three BNP values were determined in each patient. The BNP-1 was measured before surgery, BNP-2 was measured in the early post-operative phase (day 1-3), and BNP-3 was measured on the day of discharge (day 6-7). The BNP serum level was assessed by electrochemiluminescence immunoassay (Siemens, ADVIA Centauer $^{\circledR}$, USA). The measuring range provided by the manufacturer was $2-5000 \mathrm{pg} / \mathrm{mL}$. Tsubokawa and colleagues ${ }^{16}$ considered a BNP concentration of less than $20 \mathrm{pg} / \mathrm{mL}$ as normal. Sviri and associates ${ }^{11}$ collected blood samples of 10 healthy volunteers to determine a normal BNP concentration. ${ }^{11}$ According to the 2016 European Society of Cardiology guidelines for the diagnosis and treatment of acute and chronic heart failure, the normal reference value of human BNP for adults is $<35 \mathrm{pg} / \mathrm{mL}$. $^{7}$

\section{Collection of patient data}

Medical records were analyzed regarding age, sex, and comorbidities such as cardiac history, antiplatelet/anticoagulant therapy (AAT), and dementia. The time of obvious head trauma was not always possible to be determined. The modified Rankin Scale (mRS) and Glasgow Coma Scale (GCS) scores at admission, respectively, classified the functional and neurological condition of patients. Information on additional neurological impairments such as aphasia, seizure, and unconsciousness with the need for intubation before admission were collected separately.

Parameters related to post-operative complications such as rebleeding (within the first $48 \mathrm{~h}$ after surgery), myocardial infarction, pulmonary embolism, transient ischemic attack, cardiac arrest, recurrence necessitating reoperation $(\mathrm{RrR})$, postoperative ischemic stroke, ICH and death were also documented. RrR were defined as symptomatic subdural hematoma accumulation necessitating reoperation within 60 days after the first surgery.

\section{Follow-up}

Patients were followed up for three to six months after discharge in our outpatient department. The CCT scans were performed individually, dependent on the condition of the patient.

\section{Statistical analysis}

Statistical analysis was conducted using IBM SPSS Statistics ${ }^{\circledR}$ version 25 for Windows. In the descriptive analysis, continuous data are expressed with the mean and standard deviation in the case of normal distribution and with the median and the interquartile range (IQR) in the case of non-normal distribution. Categorical data are presented as frequencies and percentages.

The Wilcoxon sign-rank test was performed to assess the difference between BNP serum levels at the different stages: preoperatively, post-operatively, and at discharge. Differences between BNP-1 and categorical variables were assessed using the Mann-Whitney $U$ test. In the case of a continuous variable, the Spearman rank correlation was used. Associations between categorical variables were determined using the chi-square test and Fisher's exact test. The $p$ values $<0.05$ in two-sided testing were considered significant.

\section{Results}

\section{Patient characteristics}

In total, 100 patients were included into the current study (mean age: 73.2 years, range $42-94$ years; 78 male and 22 female patients). A flow diagram is presented in Figure 1. Patient characteristics and post-operative complications are summarized in Tables 1 and 2, respectively.

\section{Characteristics of BNP serum levels}

The BNP serum level was measured in all patients $(\mathrm{N}=100)$ preoperatively. The BNP-1 was statistically significantly elevated in $67 \%$ of the cases $(101.6$ vs. $20.9 \mathrm{pg} / \mathrm{mL}, p<0.001)$. The BNP serum level statistically significantly increased post-operatively from BNP-1 (56.65 pg/mL, IQR $107.3 \mathrm{pg} / \mathrm{mL})$ to BNP-2 $(83.50 \mathrm{pg} / \mathrm{mL}$, IQR $123.1 \mathrm{pg} / \mathrm{mL}, p<0.001)$, and then statistically significantly decreased at BNP-3 $(52.50 \mathrm{pg} / \mathrm{mL}, \mathrm{IQR} 73.5 \mathrm{pg} / \mathrm{mL}, p<0.001)$ reaching the initial level ( $p=0.264)$ (Table 3, Fig. 2). 
Table 1. Patient Characteristics

\begin{tabular}{|c|c|c|c|c|c|}
\hline Demographics and medical history & $N$ & $\%$ & Demographics and medical history & $N$ & $\%$ \\
\hline Age (mean: 73.2 , range: $42-94$ years) & & & Dementia & & \\
\hline Gender $($ male $/$ female $=3.5: 1)$ & & & *Yes & 10 & 10 \\
\hline *Female & 22 & 22 & $*$ No & 90 & 90 \\
\hline$*$ Male & 78 & 78 & Cause of cSDH & & \\
\hline Cardiac history & & & *Trauma & 80 & 80 \\
\hline *Atrial fibrillation & 13 & 13 & *Trauma not remembered & 20 & 20 \\
\hline *Coronary heart disease & 8 & 8 & $\mathrm{mRS}$ at admission & & \\
\hline *Atrial fibrillation and coronary heart disease & 7 & 7 & $* \mathrm{mRS}=0$ & 0 & 0 \\
\hline *Sick-sinus syndrome & 1 & 1 & $* \mathrm{mRS}=1$ & 9 & 9 \\
\hline *No cardiac history & 71 & 71 & $*_{\mathrm{mRS}}=2$ & 17 & 17 \\
\hline AAT & & & $*_{\mathrm{mRS}}=3$ & 21 & 21 \\
\hline$*$ ASA & 20 & 20 & $* \mathrm{mRS}=4$ & 47 & 47 \\
\hline *Phenprocoumon & 15 & 15 & $*_{\mathrm{mRS}}=5$ & 6 & 6 \\
\hline *Direct oral anticoagulants & 6 & 6 & & & \\
\hline *ASA and phenprocoumon & 3 & 3 & $* \mathrm{mRS} \leq 3$ & 47 & 47 \\
\hline *Clopidogrel & 2 & 2 & $*_{\mathrm{mRS}}>3$ & 53 & 53 \\
\hline *ASA and clopidogrel & 2 & 2 & Neurological condition at admission & & \\
\hline *No AAT & 52 & 52 & $*$ GCS score $<13$ & 13 & 13 \\
\hline Indications for AAT & & & $*$ GCS score $\geq 13$ & 87 & 87 \\
\hline *Atrial fibrillation & 18 & 18 & Aphasia & & \\
\hline *Prophylactic & 12 & 12 & *Yes & 38 & 38 \\
\hline *Coronary heart disease & 7 & 7 & $*$ No & 62 & 62 \\
\hline *History of pulmonary embolism & 4 & 4 & Seizure before admission & & \\
\hline *Dementia & 2 & 2 & *Yes & 4 & 4 \\
\hline *Bypass or Y-prothesis & 2 & 2 & $*$ No & 96 & 96 \\
\hline *History of ischemic stroke & 1 & 1 & Intubation before admission & & \\
\hline *Sick-sinus syndrome & 1 & 1 & $*$ Yes & 3 & 3 \\
\hline *Atrial fibrillation and coronary heart disease & 1 & 1 & $*$ No & 97 & 97 \\
\hline
\end{tabular}

cSDH, chronic subdural hematoma; mRS, modified Rankin scale; ASA, acetylsalicylic acid; AAT, antiplatelet/anticoagulant therapy; GCS, Glasgow Coma Scale.

Table 2. Post-Operative Complications and Functional Outcome at Discharge

\begin{tabular}{|c|c|c|}
\hline & $\mathbf{N}$ & $\%$ \\
\hline \multicolumn{3}{|l|}{ Post-operative complications } \\
\hline *Recurrence necessitating reoperation & 14 & $14.28 *$ \\
\hline *Secondary bleeding (within hospital stay) & 4 & 4 \\
\hline *Pulmonary embolism & 2 & 2 \\
\hline *Death & 2 & 2 \\
\hline *Cardiac arrest & 1 & 1 \\
\hline *Intracranial hemorrhage & 1 & 1 \\
\hline *Brain ischemia & 1 & 1 \\
\hline *No complications & 75 & 75 \\
\hline \multicolumn{3}{|l|}{$\mathrm{mRS}$ at discharge } \\
\hline$* m R S=0$ & 34 & 34 \\
\hline$* \mathrm{mRS}=1$ & 11 & 11 \\
\hline$* \mathrm{mRS}=2$ & 9 & 9 \\
\hline$* \mathrm{mRS}=3$ & 13 & 13 \\
\hline$* \mathrm{mRS}=4$ & 26 & 26 \\
\hline$* \mathrm{mRS}=5$ & 5 & 5 \\
\hline$* \mathrm{mRS}=6$ & 2 & 2 \\
\hline$* \mathrm{mRS} \leq 3$ & 67 & 67 \\
\hline$* m R S>3$ & 33 & 33 \\
\hline
\end{tabular}

mRS, modified Rankin Scale.

*of 98 patients.

\section{Patient characteristics and elevated BNP-1}

The BNP-1 was found to be statistically significantly higher in patients with atrial fibrillation $(\mathrm{AF})(\mathrm{p}<0.01)$ and patients receiving AAT $(p<0.01)$ but not in patients with a history of coronary heart disease (CHD). A statistically non-significant trend for higher BNP-1 was found in patients with dementia. Patient age and BNP-1 were statistically significantly associated in the whole cohort $(p<0.001)$ but not in the subgroup analysis $(p=0.105)$. Further, BNP elevation was independent of sex, aphasia, dysnatremia, GCS and mRS scores at admission (Table 4).

Table 3. Pre- and Post-Operative Serum Levels of Brain Natriuretic Peptide

\begin{tabular}{lccc}
\hline & \multicolumn{1}{c}{ Median $(\mathrm{pg} / \mathrm{mL})$} & $I Q R(\mathrm{pg} / \mathrm{mL})$ \\
\hline BNP-1 & 56.65 & & 107.3 \\
BNP-2 & 83.50 & & 123.1 \\
BNP-3 & 52.50 & & 73.5 \\
\hline & Median $(\mathrm{pg} / \mathrm{mL})$ & $Z$ & $\mathrm{p}$ \\
\hline BNP-1/BNP-2 & $56.65 / 83.50$ & 3.843 & $\mathbf{0 . 0 0 0 5}$ \\
BNP-2/BNP-3 & $83.50 / 52.50$ & -4.670 & $\mathbf{0 . 0 0 0 5}$ \\
BNP-1/BNP-3 & $56.65 / 52.50$ & -1.116 & 0.264 \\
\hline
\end{tabular}

$\mathrm{IQR}$, interquartile range; BNP, brain natriuretic peptide. 


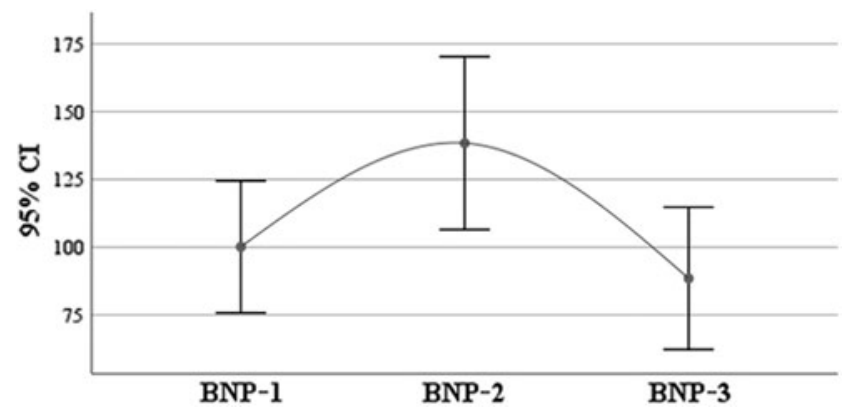

FIG. 2. Pre- and postoperative secretion pattern of brain natriuretic peptide (BNP) in patients with symptomatic chronic subdural hematoma. CI, confidence interval.

\section{Characteristics of pre- and post-operative $m R S$ scores and their relationship with BNP-1}

The proportion of patients with favorable versus unfavorable mRS score at discharge was statistically significantly different from that at admission (McNemar test; $p<0.001$ ). Indeed, 24 patients with an initially unfavorable $\mathrm{mRS}$ score post-operatively improved and had a good outcome at discharge; in contrast, only four patients with an initially favorable $\mathrm{mRS}$ score post-operatively stagnated/deteriorated and had a poor outcome at discharge (Fig. 3). Using the Mann-Whitney $U$ test, median BNP-1 was statistically significantly higher in patients with unfavorable versus favorable $\mathrm{mRS}$ score at discharge ( $81.3 \mathrm{vs.} 45 \mathrm{pg} / \mathrm{mL}, p=0.014)$, in contrast to that at admission (64.8 vs. $43 \mathrm{pg} / \mathrm{mL}, p=0.064)$.

\section{Predictors of $m R S$ score at discharge}

Univariate analysis revealed that elevated BNP-1 was statistically significantly associated with a poor outcome at discharge $(\mathrm{OR}=3.038, p=0.041)$. After adjusting for several confounders of mRS score at discharge such as patient age, presence of dementia, GCS and mRS scores at admission, the multivariate analysis revealed that BNP-1 was not able to predict the functional outcome at discharge (Table 5).

\section{Discussion}

The current study is the first to investigate the pre- and postoperative secretion pattern of BNP serum levels in patients with
cSDH. In addition, it assesses the relationship between preoperative BNP and patient demographics, medical history, neurological condition, and functional outcome at discharge. We found that pre-operative BNP serum levels were significantly increased in $67 \%$ of patients with symptomatic cSDH. The BNP concentrations increased rapidly after surgery, then decreased at discharge to nearly the initial pre-operative level. Moreover, elevated BNP serum levels were significantly higher in patients with $\mathrm{AF}$ and patients receiving AAT but not in patients with a history of CHD.

Although elevated pre-operative BNP concentrations were statistically significantly associated with a poor outcome at discharge, after adjusting for several confounders (age, dementia, mRS and GCS scores at admission), BNP-1 was not able to predict poor outcome at discharge. Finally, there was no association between the pre-operative BNP plasma concentrations and hyponatremia.

Previous studies demonstrated that the BNP plasma concentration was increased in several acute pathologies of the CNS. In patients with aSAH, this elevation was positively associated with the severity of the clinical onset (World Federaton of Neurologic Surgeons Grade V) and the location of the ruptured aneurysm at the anterior communicating artery. ${ }^{16}$ Indeed, the mechanical stress of the anterior hypothalamus through the blood jet after aneurysm rupture would explain this hypersecretion of BNP. ${ }^{14}$ Further, the release of atrial BNP may be part of a general stress response to surgery or intensive care treatment. ${ }^{14}$ This phenomenon was observed post-operatively in our cohort of patients.

In other reports, elevated BNP plasma concentration was associated with neurogenic stress cardiomyopathy after aSAH and exhibited a poor prognostic power. ${ }^{17}$ Other authors described an association of high BNP levels and the occurrence of delayed ischemic neurological deficits in patients with aSAH. ${ }^{15,18}$ Recently, the secretion pattern of N-terminal (NT)-proBNP, a non-active prohormone that is released from the same molecule that produces $\mathrm{BNP}$, was assessed in patients with hypertensive ICH.

It was also demonstrated, that BNP serum levels were elevated in the acute phase of cerebral infarction and declined in the subacute phase. ${ }^{8,9,25}$ A previous report affirmed that an increased BNP level in these patients was a risk factor for thromboembolic events. ${ }^{9}$ In other reports, the BNP serum level could significantly distinguish between cardioembolic stroke and other stroke subtypes. ${ }^{10}$ In contrast, increased BNP concentrations in patients with TBI were associated with increased ICP, ${ }^{11-13}$ hyponatremia, ${ }^{13}$ the severity of the trauma, ${ }^{13}$ and a poor outcome. ${ }^{11}$ Analogous to these observations, in which elevated BNP secretion occurs in the acute phase of

Table 4. Patient Characteristics and Elevated Brain Natriuretic Peptide Serum Levels

\begin{tabular}{|c|c|c|c|c|c|c|}
\hline \multirow{2}{*}{$\begin{array}{l}\text { Variables } \\
\text { Mann-Whitney U test }\end{array}$} & \multicolumn{6}{|c|}{$B N P-1 \geq 35 \mathrm{pg} / \mathrm{mL}$} \\
\hline & $N$ & Medians $(\mathrm{pg} / \mathrm{mL})$ & $U$ & $Z$ & Corr. coef. $r$ & $\mathrm{p}$ \\
\hline Age (Spearman rank correlation) & 67 & - & - & - & 0.200 & 0.105 \\
\hline Gender & 67 & Female (101.6) / Male (99.5) & 437 & -0.622 & - & 0.534 \\
\hline Atrial fibrillation & 67 & No $(69.1) /$ Yes $(174.7)$ & 715 & 3.876 & - & 0.0005 \\
\hline Coronary heart disease & 67 & No (86.1) / Yes (141.3) & 364 & 1.390 & - & 0.165 \\
\hline Antiplatelet/anticoagulant therapy & 67 & No $(60.2)$ / Yes (136.1) & 826 & 3.480 & - & 0.001 \\
\hline $\mathrm{mRS}$ at admission (cut-off 3 ) & 67 & $\leq 3(107.9) />3(96.3)$ & 567 & 0.352 & - & 0.725 \\
\hline Dementia & 67 & No $(88.5) /$ Yes $(183.0)$ & 326 & 1.740 & - & 0.082 \\
\hline Aphasia at admission & 67 & No (88.5) / Yes (116.1) & 549 & 0.721 & - & 0.471 \\
\hline GCS score at admission (cut-off 13 ) & 67 & $\leq 13(126.4) />13(91.1)$ & 274.5 & 0.744 & - & 0.457 \\
\hline
\end{tabular}

BNP, brain natriuretic peptide; mRS, modified Rankin Scale; GCS, Glasgow Coma Scale. 


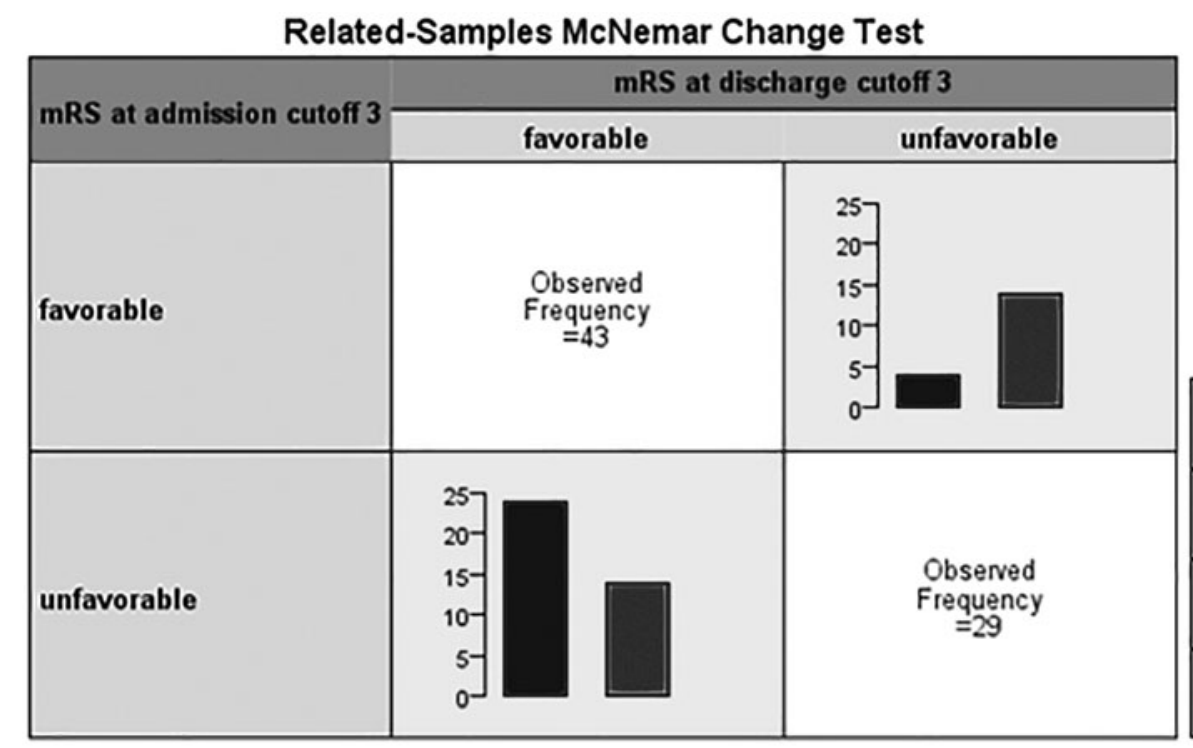

\section{Frequency \\ Observed \\ Hypothesized}

\begin{tabular}{|lr|}
\hline Total N & 100 \\
\hline Test Statistic & 12,893 \\
\hline Degrees of Freedom & 1 \\
\hline Asymptotic Sig. (2-sided test) & .000 \\
\hline
\end{tabular}

FIG. 3. McNemar Test showing a statistically significant difference in the proportions of patients with favorable versus unfavorable modified Rankin Scale (mRS) scores at admission and discharge.

Table 5. Predictors of Functional Outcome AT Discharge

Univariate Analysis

\begin{tabular}{lcccccc}
\hline \multirow{2}{*}{ Variables } & \multicolumn{5}{c}{$m R S$ at discharge } \\
\cline { 3 - 6 } Chi-square test & $N$ & $\leq 3$ & $>3$ & OR & $\mathrm{p}$ \\
\hline Gender (female) & 100 & $13 / 67$ & $9 / 33$ & - & 0.443 \\
Cardiac history & 100 & $18 / 67$ & $11 / 33$ & - & 0.640 \\
AAT & 100 & $30 / 67$ & $18 / 33$ & - & 0.400 \\
mRS at admission $(>3)$ & 100 & $24 / 67$ & $29 / 33$ & $\mathbf{1 2 . 9 9 0}$ & $\mathbf{0 . 0 0 0 5}$ \\
Dementia & 100 & $3 / 67$ & $7 / 33$ & $\mathbf{5 . 7 4 4}$ & $\mathbf{0 . 0 1 4}$ \\
Aphasia at admission & 100 & $23 / 67$ & $15 / 33$ & - & 0.381 \\
Presence of trauma & 100 & $54 / 67$ & $26 / 33$ & - & 1.000 \\
GCS at admission $(<13)$ & 100 & $5 / 67$ & $8 / 33$ & $\mathbf{3 . 9 6 8}$ & $\mathbf{0 . 0 2 7}$ \\
BNP-1 $(\geq 35$ pg/ml) & 100 & $40 / 67$ & $27 / 33$ & $\mathbf{3 . 0 3 8}$ & $\mathbf{0 . 0 4 1}$
\end{tabular}

\begin{tabular}{lccc}
\hline $\begin{array}{c}\text { Spearman rank } \\
\text { correlation }\end{array}$ & $N$ & Corr. coef. $r$ & $\mathrm{p}$ \\
\hline Age & 100 & 0.331 & $\mathbf{0 . 0 0 1}$ \\
BNP-1 & 100 & 0.246 & $\mathbf{0 . 0 1 4}$ \\
\hline
\end{tabular}

Multivariate Analysis

\begin{tabular}{lccc}
\hline $\begin{array}{l}\text { Binomial logistic } \\
\text { regression }\end{array}$ & \multicolumn{3}{c}{ mRS at discharge } \\
\hline Predictors & $B$ & $\operatorname{Exp}(B)$ & $\mathrm{p}$ \\
\hline mRS at admission $(>3)$ & 2.613 & 13.638 & $\mathbf{0 . 0 0 0 5}$ \\
Age & 0.069 & 1.071 & $\mathbf{0 . 0 1 0}$ \\
BNP-1 & - & - & 0.192 \\
GCS at admission $(<13)$ & - & - & 0.155 \\
Dementia at admission & - & - & 0.139 \\
\hline
\end{tabular}

mRS, modified Rankin Scale; OR, odds ratio; AAT, antiplatelet/anticoagulant therapy; GCS. Glasgow Coma Scale; BNP, brain natriuretic peptide. the CNS diseases, our findings suggest that BNP hypersecretion, which was observed in $67 \%$ of the patients with symptomatic $\mathrm{cSDH}$, may be related to the acute onset of neurological symptoms because of brain compression.

The secretion pattern of BNP in the serum has been assessed in many studies including in patients with CNS diseases. In patients with ICH, the peak-to-mean concentration of NT-proBNP was reached on day 4 , followed by reduction to the initial level by day 7 . Thereafter, the plasma levels continued to decline. ${ }^{19}$ The same secretion pattern was observed in patients with TBI, where the peak-to-mean concentration occurred on day $3 .{ }^{13}$ Our findings are in line with these observations, as the peak-to-mean concentration of BNP occurred between post-operative days 1 and 3. In our cohort, however, this elevation could be explained through a general response to surgery. In addition, the BNP serum level at discharge (on day 6 or 7) did not significantly differ from that on admission.

Other authors have reported a delayed peak-to-mean concentration of BNP in patients with TBI (on day 7), which has been suggested as being dependent on the relatively slow replacement of sodium loss in those patients. ${ }^{11}$ In patients with aSAH, two peaks of BNP levels were observed after clipping (on the second and fourth day). ${ }^{14}$

Significantly higher levels of BNP were reported in the acute phase of stroke in patients with $\mathrm{AF}^{8-10}$ which might reflect the greater severity of left atrial dilation and cardiac hypertrophy. ${ }^{8}$ In our cohort of cSDH patients, our findings were in line with these observations. In addition, a statistically significantly higher concentration was observed in patients receiving AAT, as all patients with $\mathrm{AF}$ were receiving this treatment. Patients with a history of CHD, however, did not show any significant difference in BNP levels compared with patients without CHD.

Although the association of BNP concentrations with age is already known, ${ }^{9,26}$ our findings showed no statistically significant association in the subgroup analysis, probably because of the small size of our cohort. In contrast, the presence of dementia revealed a statistically non-significant trend $(p=0.082)$, probably because of the coexistence of $\mathrm{AF}$ in $50 \%$ of these patients.

In several studies, the BNP serum level at admission was used as a predictor of functional outcome. Indeed, stroke patients with 
initially elevated BNP levels had poor outcomes at discharge, if the time of BNP measurement was within $24 \mathrm{~h}$ of stroke onset. ${ }^{27}$ In our study, increased pre-operative BNP serum levels were also statistically significantly associated with a poor outcome at discharge; nevertheless, this result was statistically not significant in the multivariate analysis.

A BNP-cutoff value of $89 \mathrm{pg} / \mathrm{mL}$ was used as a predictor of a good outcome $(\mathrm{mRS} \leq 2)$ at 90 days after stroke. ${ }^{25}$ Further, the NTproBNP serum level was an independent predictor of death and poor outcome at three months in patients with low-risk nontraumatic $\mathrm{ICH}^{28}$ Admission BNP level in acute ischemic stroke patients receiving intravenous thrombolysis could not predict the outcome at three months, however. ${ }^{29}$ In addition, high serum levels of BNP could not predict functional outcome in patients with aSAH,${ }^{30}$ but a decreasing BNP level predicted a significant improvement in GCS score over a two-week period. ${ }^{15}$ In patients with TBI, increased BNP plasma concentrations were significantly associated with a poor outcome. ${ }^{11}$

In contrast to these findings, the onset of symptoms is often subtle in patients with symptomatic $\mathrm{cSDH}$, in which BNP serum levels are frequently collected a few days to a few weeks after the onset of symptoms. Whether long-term functional outcome (3-6 months after surgery) could be predicted is a further end point of this ongoing prospective study.

The BNP plays an important role in the regulation of the waterelectrolyte balance, and its hypersecretion was demonstrated to be significantly associated with the occurrence of aSAH, ${ }^{15}$ severe TBI, ${ }^{13}$ and severe $\mathrm{ICH}^{19}$ but not with acute ischemic stroke. ${ }^{8}$ In our cohort, we found no association between BNP serum levels and hyponatremia.

Altogether, the increase of the BNP serum levels in the early post-operative period is probably because of cardiac stress induced by surgery through local manipulation of the hematoma. In this way, elevated pre-operative BNP concentrations might be the effect of cardiac stress from the increasing brain compression by the hematoma-i.e., elevation of the ICP.

Recently, a brain-heart crosstalk was described in patients with TBI who frequently experienced paroxysmal sympathetic hyperactivity after trauma, also known as "autonomic dysfunction," leading to cardiac wall abnormalities and dysfunction. ${ }^{31}$ Other authors demonstrated that elevated ICP in these patients caused increased sympathetic activity and catecholamine hypersecretion. ${ }^{32}$

Although there is still a knowledge gap regarding the mechanisms of autonomic dysfunction, rat models already revealed that post-traumatic injury of the hypothalamus, ${ }^{33}$ increase in the activity of the hypothalamic-sympathetic-adrenal-medullary axis, ${ }^{34}$ and circulating pro-inflammatory cytokines ${ }^{35}$ play a role in autonomic and cardiovascular dysfunction. Whether a dysregulation of the autonomic nervous system is responsible for the increase in pre-operative BNP concentrations in $\mathrm{CSDH}$ should be further investigated.

\section{Limitations of the study}

We attempted to exclude all factors that may have contributed to the increased BNP serum levels (CHF, history of cardiac infarction, renal insufficiency, etc.). In addition, among the 29 eligible patients without $\mathrm{CHF}$, but with a history of $\mathrm{AF}$ and/or $\mathrm{CHD}$, where $\mathrm{BNP}$ serum levels were elevated, echocardiography was performed preoperatively in the patients with a history of CHD $(n=15)$ revealing a normal ejection fraction (EF) of the left ventricle. The eventual presence of a not yet diagnosed heart failure with preserved EF and so with non-specific signs and symptoms ${ }^{7}$ was not pre-operatively investigated, however. Further, the small sample size of the study population may not represent accurately the wide spectrum of patients with cSDH.

Moreover, most of the patients could not remember exactly if they had a trauma in the last days/weeks or report the approximate date of the trauma may be because of the banality of the event and/or the presence of a cognitive impairment (dementia, change in behavior, age-associated memory impairment). Thus, we could not precisely estimate the duration of the neurological symptoms by each patient and their exact time of onset, which might be helpful to further explain the different BNP serum levels at admission.

\section{Conclusion}

The current study provides new evidence regarding the BNP serum level and secretion pattern in patients with symptomatic cSDH and creates a paradigm for future studies. The BNP plasma concentrations are elevated frequently. Indeed, they increase significantly after surgery, then decrease to reach the initial level. Whether BNP serum levels can be used to predict long-term functional outcome is a further end point of this ongoing prospective study.

\section{Authors' Contributions}

Dr. Chihi helped conceptualizing and designing the study, acquired, analyzed, and interpreted the data, drafted the initial manuscript, and reviewed and revised the final version of the manuscript. Drs. Helsper, Gembruch, Darkwah Oppong, Jabbarli. and Wrede analyzed and interpreted the data, and reviewed and revised the final version of the manuscript. Dr. Huetter contributed in the study with his expertise in statistics and revised the final version of the manuscript. Prof. Sure revised the final version of the manuscript. Dr. Maslehaty conceptualized and designed the study, analyzed and interpreted the data, revised the manuscript for important intellectual content, and reviewed and revised the final version of the manuscript.

All authors approved the final manuscript as submitted and agree to be accountable for all aspects of the work.

\section{Funding Information}

No funding was secured for this study.

\section{Author Disclosure Statement}

No competing financial interests exist.

\section{References}

1. Abdelalim, E.M., Takada, T., Torii, R., and Tooyama, I. (2006). Molecular cloning of BNP from heart and its immunohistochemical localization in the hypothalamus of monkey. Peptides 27, 1886-1893.

2. Sudoh, T., Kangawa, K., Minamino, N., and Matsuo, H. (1988). A new natriuretic peptide in porcine brain. Nature 332, 78-81.

3. Pandey, K.N. (2005). Biology of natriuretic peptides and their receptors. Peptides 26, 901-932.

4. Espiner, E.A. (1994). Physiology of natriuretic peptides. Intern. Med. 235, 527-541.

5. Takahashi, K., Totsune, K., Sone, M., Ohneda, M., Murakami, O., Itoi, K., and Mouri, T. (1992). Human brain natriuretic peptide-like immunoreactivity in human brain. Peptides 13, 121-123.

6. Porzionato, A., Macchi, V., Rucinski, M., Malendowicz, L.K., and De Caro, R. (2010). Natriuretic peptides in the regulation of the hypothalamic-pituitary-adrenal axis. Int. Rev. Cell Biol. 280, 1-39. 
7. Ponikowski, P., Voors, A.A., Anker, S.D., Bueno, H., Cleland, J.G. Coats, A.J., Falk, V., González-Juanatey, J.R., Harjola, V.P., Jankowska, E.A., Jessup, M., Linde, C., Nihoyannopoulos, P., Parissis, J.T., Pieske, B., Riley, J.P., Rosano, G.M., Ruilope, L.M., Ruschitzka, F., Rutten, F.H., and van der Meer, P. (2016). 2016 ESC Guidelines for the diagnosis and treatment of acute and chronic heart failure: The Task Force for the diagnosis and treatment of acute and chronic heart failure of the European Society of Cardiology (ESC). Developed with the special contribution of the Heart Failure Association (HFA) of the ESC. Eur. J. Heart Fail. 18, 891-975.

8. Nakagawa, K., Yamaguchi, T., Seida, M., Yamada, S., Imae, S., Tanaka, Y., Yamamoto, K. and Ohno, K. (2005). Plasma concentrations of brain natriuretic peptide in patients with acute ischemic stroke. Cerebrovasc. Dis. 19, 157-164.

9. Di Angelantonio, E., De Castro, S., Toni, D., Sacchetti, M.L., Biraschi, F., Prencipe, M. and Fiorelli, M. (2007). Determinants of plasma levels of brain natriuretic peptide after acute ischemic stroke or TIA. J. Neurol. Sci. 260, 139-142.

10. Montaner, J., Perea-Gainza, M., Delgado, P., Ribó, M., Chacón, P., Rosell, A., Quintana, M., Palacios, M.E., Molina, C.A., and AlvarezSabín, J. (2008). Etiologic diagnosis of ischemic stroke subtypes with plasma biomarkers. Stroke 39, 2280-2287.

11. Sviri, G., Soustiel, J., and Zaaroor, M. (2006). Alteration in brain natriuretic peptide (BNP) plasma concentration following severe traumatic brain injury. Acta Neurochir. 148, 529-533.

12. Kirchhoff, C., Stegmaier, J., Bogner, V., Buhmann, S., Mussack, T., Kreimeier, U., Mutschler, W., and Biberthaler, P. (2006). Intrathecal and systemic concentration of NT-proBNP in patients with severe traumatic brain injury. J. Neurotrauma 23, 943-949.

13. Wu, X., Sha, H., Sun, Y., Gao, L., Liu, H., Yuan, Q., Zhang, T., Zhu, J., Zhou, L., and Hu, J. (2011). N-terminal pro-B-type natriuretic peptide in patients with isolated traumatic brain injury: a prospective cohort study. J. Trauma 71, 820-825.

14. Berendes, E., Walter, M., Cullen, P., Prien, T., Van Aken, H., Horsthemke, J., Schulte, M., von Wild, K., and Scherer, R. (1997). Secretion of brain natriuretic peptide in patients with aneurysmal subarachnoid haemorrhage. Lancet 349, 245-249.

15. McGirt, M.J., Blessing, R., Nimjee, S.M., Friedman, A.H., Alexander, M.J., Laskowitz, D.T., and Lynch, J.R. (2004). Correlation of serum brain natriuretic peptide with hyponatremia and delayed ischemic neurological deficits after subarachnoid hemorrhage. Neurosurgery 54 , 1369-1373.

16. Tsubokawa, T., Shiokawa, Y., Kurita, H., and Kaneko, N. (2004). High plasma concentration of brain natriuretic peptide in patients with ruptured anterior communicating artery aneurysm. Neurol. Res. 26, 893-896.

17. Tung, P.P., Olmsted, E., Kopelnik, A., Banki, N.M., Drew, B.J., Ko, N., Lawton, M.T., Smith, W., Foster, E., Young, W.L., and Zaroff, J.G. (2005). Plasma B-type natriuretic peptide levels are associated with early cardiac dysfunction after subarachnoid hemorrhage. Stroke 36, 1567-1569.

18. Jabbarli, R., Pierscianek, D., Oppong, M.D., Sato, T., Dammann, P., Wrede, K.H., Kaier, K., Köhrmann, M., Forsting, M., Kleinschnitz, C., Roos, A., and Sure, U. (2020). Laboratory biomarkers of delayed cerebral ischemia after subarachnoid hemorrhage: a systematic review. Neurosurg. Rev. 43, 825-833.

19. Li, F., Chen, Q.X., Xiang, S.G., Yuan, S.Z., and Xu, X.Z. (2018). $\mathrm{N}$-Terminal pro-brain natriuretic peptide concentrations after hypertensive intracerebral hemorrhage: relationship with hematoma size hyponatremia, and intracranial pressure. J. Intensive Care Med. 33, 663-670.

20. Miranda, L.B., Braxton, E., Hobbs, J., and Quigley, M.R. (2011). Chronic subdural hematoma in the elderly: not a benign disease. J. Neurosurg. 114, 72-76

21. Lee, K.S., Bae, W.K., Doh, J.W., Bae, H.G., and Yun, I.G. (1998). Origin of chronic subdural haematoma and relation to traumatic subdural lesions. Brain Inj. 12, 901-910.
22. Astrup, T. (1965). Assay and content of tissue thromboplastin in different organs. Thromb. Diath. Haemorrh. 14, 401-416.

23. Edlmann, E., Giorgi-Coll, S., Whitfield, P.C., Carpenter, K., and Hutchinson, P. (2017). Pathophysiology of chronic subdural haematoma: inflammation, angiogenesis and implications for pharmacotherapy. J. Neuroinflammation 14, 108.

24. Stanišić, M., and Pripp, A.H. (2017). A reliable grading system for prediction of chronic subdural hematoma recurrence requiring reoperation after initial burr-hole surgery. Neurosurgery 81, 752-760.

25. Chaudhuri, J.R., Sharma, V.K., Mridula, K.R., Balaraju, B., and Bandaru, V.C. (2015). Association of plasma brain natriuretic peptide levels in acute ischemic stroke subtypes and outcome. J. Stroke Cerebrovasc. Dis. 24, 485-491.

26. Galinier M. BNP. NT-proBNP: lequel choisir en pratique? Réalités cardiologiques. 2010. https://www.realites-cardiologiques.com/wpcontent/uploads/sites/2/2010/11/091.pdf (Last accessed June 22, 2020).

27. Fukuhara, K., Ogata, T., Takeshita, S., and Tsuboi, Y. (2019). Serum B-type natriuretic peptide level and timing of its measurement as a predictor of acute ischemic stroke outcome. eNeurologicalSci. 18, 100217.

28. Gregorio, T., Albuquerque, I., Neves, V., Reinas, R., Pipa, S., Azevedo, L., and Chaves, P.C. (2019). NT-pro-BNP correlates with disease severity and predicts outcome in cerebral haemorrhage patients:cohort study. J. Neurol. Sci. 399, 51-56.

29. Gupta, H.V., Finlay, C.W., Jacob, S., Raina, S.K., Lee, R.W., and Hinduja, A. (2019). Can admission BNP level predict outcome after intravenous thrombolysis in acute ischemic stroke? Neurologist 24, 6-9.

30. McAteer, A., Hravnak, M., Chang, Y., Crago, E.A., Gallek, M.J., and Yousef, K.M. (2017). The relationships between BNP and neurocardiac injury severity, noninvasive cardiac output, and outcomes after aneurysmal subarachnoid hemorrhage. Biol Res. Nurs. 19, 531-537.

31. Khalid, F., Yang, G.L., McGuire, J.L., Robson, M.J., Foreman, B. Ngwenya, L.B., and Lorenz, J.N. (2019). Autonomic dysfunction following traumatic brain injury: translational insights. Neurosurg. Focus 47, E8.

32. Belzberg, H., Shoemaker, W.C., Wo, C.C., Nicholls, T.P., Dang, A.B., Zelman, V., Gruen, J.P., Berne, T.V., and Demetriades, D. (2007). Hemodynamic and oxygen transport patterns after head trauma and brain death: implications for management of the organ donor. J. Trauma 63, 1032-1042.

33. Nosaka, S. (1966). Hypertension induced by extensive medial anteromedian hypothalamic destruction in the rat. Jpn. Circ. J. 30, 509523.

34. Griesbach, G.S., Hovda, D.A., Tio, D.L., and Taylor, A.N. (2011). Heightening of the stress response during the first weeks after a mild traumatic brain injury. Neuroscience 178, 147-158.

35. Wei, S.G., Zhang, Z.H., Beltz, T.G., Yu, Y., Johnson, A.K., and Felder, R.B. (2013). Subfornical organ mediates sympathetic and hemodynamic responses to blood-borne proinflammatory cytokines. Hypertension 62, 118-125.

Address correspondence to: Mehdi Chihi, MD Department of Neurosurgery and Spine Surgery University Hospital Essen University of Duisburg-Essen Hufelandstrasse 55 45147 Essen Germany

E-mail: mehdi.chihi@uk-essen.de 